\title{
The prognostic factors of effective ketoconazole treatment for metastatic castration-resistant prostate cancer: who can benefit from ketoconazole therapy?
}

\author{
Guo-Wen Lin ${ }^{1,2, *}$, Xu-Dong Yao ${ }^{1,2, *}$, Ding-Wei Ye ${ }^{1,2}$, Yao Zhu ${ }^{1,2}$, Shi-Lin Zhang ${ }^{1,2}$, Bo Dai ${ }^{1,2}$, Hai-Liang Zhang ${ }^{1,2}$, \\ Yi-Jun Shen ${ }^{1,2}$ and Chun-Guang $\mathrm{Ma}^{1,2}$
}

We investigated the prognostic value of some variables of effective ketoconazole treatment for metastatic castration-resistant prostate cancer (mCRPC). In total, 163 patients with mCRPC were eligible, receiving ketoconazole 200-400 mg three times daily with replacement doses of prednisone. Progression-free survival (PFS) was calculated from the beginning of the ketoconazole therapy to the onset of disease progression. The prognostic value of different variables for PFS was assessed by Cox regression analysis. The median PFS was 2.6 months ( $0.5-8.6$ months) for these patients. The serum testosterone level changed during therapy, which decreased when the prostate-specific antigen (PSA) declined; the serum testosterone level increased as the levels of PSA relapsed. The median PFS values for patients associated with different factors were the following: 1.4 and 3.5 months for a nadir PSA of $\geqslant 0.2$ and $<0.2 \mathrm{ng} \mathrm{ml}^{-1}$, respectively (hazard rate (HR)=4.767, $P<0.001$ ); 3.1 and 1.6 months for a baseline testosterone of $\geqslant 0.1$ and $<0.1 \mathrm{ng} \mathrm{ml}^{-1}$, respectively (HR=2.865, $P=0.012$ ); 2.8 and 1.9 months for a baseline haemoglobin of $\geqslant 120$ and $<120 \mathrm{~g} \mathrm{I}^{-1}$, respectively (HR=1.605, $P<0.001$ ); and 3.0 and 1.9 months for a PSA doubling time (PSADT) of $\geqslant 2.0$ and $<2.0$ months, respectively ( $H R=1.454, P=0.017)$. A risk model was constructed according to the four factors that divided patients into three subgroups of low risk (0-1 factors), moderate risk (2 factors) and high risk (3-4 factors) with PFS values of 3.6, 3.0 and 1.4 months, respectively (HR=1.619, $P<0.001$ ). A nadir PSA of $\geqslant 0.2 \mathrm{ng} \mathrm{ml}^{-1}$, a baseline testosterone of $<0.1 \mathrm{ng} \mathrm{ml}^{-1}$, a baseline haemoglobin of $<120 \mathrm{~g} \mathrm{I}^{-1}$ and a PSADT of $<2$ months were associated with a poor PFS. This risk model could provide evidence to predict the survival benefit of ketoconazole therapy.

Asian Journal of Andrology (2012) 14, 732-737; doi:10.1038/aja.2012.57; published online 20 August 2012

Keywords: castration-resistant prostate cancer; ketoconazole therapy; predictor; progression-free survival

\section{INTRODUCTION}

Although hormonal therapy has been introduced for nearly 60 years, it is still the first treatment choice for patients with advanced prostate cancer, conferring an improvement in the quality of life and survival benefit. ${ }^{1,2}$ Although hormonal therapy is effective as an initial treatment, it is not a cure for prostate cancer. Most prostate cancer inevitably develops into a hormone-resistant disease with prostate-specific antigen (PSA) relapse. The treatment options for castration-resistant prostate cancer (CRPC) are limited, including anti-androgen changes, oestrogenic therapy, other androgen synthesis inhibitors (such as ketoconazole or abiraterone) and chemotherapy. ${ }^{3-6}$ Of all these treatments, ketoconazole shows promising results of slowing the disease progression with moderate toxicity and economic burden. ${ }^{7-9}$

Ketoconazole is commonly used as an antifungal drug, and it can inhibit adrenal testosterone synthesis as inhibitor of cytochrome P450 $14 \alpha$-demethylase (CYP17). ${ }^{7}$ Ketoconazole 200 or 400 mg three times daily is given as a subsequent hormonal manipulation for patients with
CRPC. Approximately $30 \%-60 \%$ of patients with CRPC have a serum PSA decline of $\geqslant 50 \%$ and a progression-free survival (PFS) of 3.2-8.6 months. ${ }^{7,10,11}$ The single largest prospective study of ketoconazole, the CALGB9583 trial, enrolled 260 cases treated with simultaneous antiandrogen withdrawal and ketoconazole after the failure of hormonal therapy and indicated that $27 \%$ patients had a PSA decline of $\geqslant 50 \%$ with a PFS of 8.6 months. ${ }^{11}$ Furthermore, ketoconazole-combined chemotherapy is another therapeutic option for patients with CRPC, and the PSA response rates are $25 \%$ and $70 \%$ for the docetaxel-based and mitoxantrone-based chemotherapy, respectively. ${ }^{12,13}$

In most reported studies, ${ }^{7,12,13}$ the PFS and PSA response rates of ketoconazole therapy are only moderate, meaning that not all the patients will benefit from the ketoconazole therapy. However, few studies have explored the predictors for ketoconazole effective treatment. In one clinical trial, androstenedione was found to be a predictor for the patients treated with ketoconazole therapy, but controversy still exists because another study denied the association 
between androstenedione and the response to ketoconazole therapy. ${ }^{14,15}$ Given the mechanism of ketoconazole as a CYP17 inhibitor of adrenal testosterone synthesis, there might be some relationship between testosterone and the outcome of ketoconazole therapy. In this retrospective study, we tried to explore the possible prognostic factors of ketoconazole therapy for patients with metastatic CRPC (mCRPC) and found who could benefit from ketoconazole therapy.

\section{MATERIALS AND METHODS}

\section{Patients}

A total of 163 patients with metastatic prostate adenocarcinoma were identified as eligible candidates from May 2006 to April 2011 at our hospital. Androgen-deprivation therapy, including orchiectomy or luteinizing hormone-releasing hormone agonist, with an anti-androgen as the initial hormonal therapy, was applied beginning from the primary diagnosis. When the disease progressed, the patients were treated with anti-androgen withdrawal for 4-6 weeks and subsequent hormonal therapy (anti-androgen changes and an oestrogenic agent), but their PSA continuously increased and all the patients entered the CRPC stage. The definition of CRPC met the criterion in the European Association of Urology Guidelines 2011. All the patients had lymph node, bone or internal organ metastasis, except brain metastasis, at the CRPC stage. The patients were excluded if they had received prior ketoconazole therapy, corticosteroids, aminoglutethimide or experimental therapy. Chemotherapy before the ketoconazole therapy was permitted. The eligible subjects had adequate organ function with a life expectancy of more than 3 months. ${ }^{11}$

\section{Treatment}

The enrolled patients were given ketoconazole $200 \mathrm{mg}$ orally three times daily post cibum with prednisone $5 \mathrm{mg}$ orally twice a day until the PSA continued to increase or the treatment resulted in unacceptable toxicity as described below. Ketoconazole $400 \mathrm{mg}$ was considered after ketoconazole $200 \mathrm{mg}$ was found to be a failure for these cases. The luteinizing hormone-releasing hormone agonist was continuously given during the ketoconazole therapy. All patients came to the hospital for an evaluation every month during the ketoconazole therapy. The symptoms were assessed, a physical examination was performed, a medical history was recorded, and an imaging examination, including computed tomography, of the abdomen and pelvis, a bone scan and a chest radiograph were made if needed. The patients' plasma samples were collected at approximately 8:00 a.m. at the baseline assessment and monthly thereafter. The treatment-related toxicity was assessed using the National Cancer Institute Common Toxicity Criteria for Adverse Events version 3.0. If grade-3 or grade- 4 toxicity was present, the treatment was stopped; if less than grade 3 , the patients were monitored for toxicity, and this management was continued until disease progression.

\section{Statistical analysis}

The data in this study included the following variables: age, clinical T stage, PSA, biopsy Gleason score, previous anti-androgen therapy, PSA doubling time (PSADT) and baseline testosterone, haemoglobin and alkaline phosphatase during the ketoconazole therapy. The nadir PSA was defined as the nadir PSA during the previous treatments before the ketoconazole therapy. The PSADT was calculated as the PSA kinetics over time from the nadir PSA to the baseline PSA. The median follow-up duration was 51.9 months (9.0-127.4 months).

According to the PSA Working Group Consensus Criteria, the PSA response rate was defined as the percentage of patients with a PSA decline of $\geqslant 50 \%$ compared with the baseline PSA level during the ketoconazole therapy. ${ }^{16}$ The primary endpoint was defined as either the date the PSA increased to $50 \%$ above the nadir if a $\geqslant 50 \%$ decline in PSA had been reached, the date the PSA increased 25\% above the nadir if a $<50 \%$ decline had been reached, the date the PSA increased $25 \%$ above the baseline if no PSA decline had been experienced or the bone/soft tissue metastasis had progressed by an imaging test. ${ }^{7,16}$ The PFS was calculated from the beginning of the ketoconazole therapy to the primary endpoint.

The relationships between the serum PSA levels and testosterone levels during the ketoconazole therapy were analysed using Spearman's rank correlations. The univariate and multivariate Cox regression analyses were used to assess the prognostic value of the variables and develop the risk model for survival. The median PFS was computed using the Kaplan-Meier method. The differences in the PFS were assessed by a log-rank test. The cutoff points for the nadir PSA and PSADT were $0.2 \mathrm{ng} \mathrm{ml}^{-1}$ and 2.0 months, respectively, which were based on the literature. ${ }^{17,18}$ The cutoff points for the baseline PSA and testosterone during the ketoconazole therapy were 20 and $0.1 \mathrm{ng} \mathrm{ml}^{-1}$, respectively, because they had the greatest prognostic values according to the Youden index, which was a good measure of diagnostic effectiveness that was calculated as sensitivity + specificity $-1 .{ }^{19}$ Potential predictive variables were considered to be risk factors, by which the patients were divided into risk subgroups for further survival analysis. A $P$ value of $<0.05$ was considered to be a statistically significant difference. All statistical calculations were computed using SPSS (Statistical Package for the Social Sciences) version 13.0 (SPSS Inc., Chicago, IL, USA).

\section{RESULTS}

\section{Patient characteristics}

The clinical and pathological characteristics for the 163 enrolled patients are shown in Table 1. Eleven patients received chemotherapy with docetaxel, and three received oestrogen therapy. The median values for the nadir PSA, PSADT, baseline PSA, testosterone, haemoglobin and alkaline phosphatase were $2.8 \mathrm{ng} \mathrm{ml}^{-1}, 2.3$ months, $21.0 \mathrm{ng} \mathrm{ml}^{-1}, 0.12 \mathrm{ng} \mathrm{ml}^{-1}, 117 \mathrm{~g} \mathrm{l}^{-1}$ and $171 \mathrm{IU} \mathrm{l}^{-1}$, respectively. There were some missing data for the clinical $\mathrm{T}$ stage (64 cases lost) because some information was missing from the record at diagnosis. The median duration of the previous hormonal therapy before the ketoconazole therapy was 23.3 months (7.1-107.1 months).

In this study, we were interested in the change of the serum testosterone during ketoconazole therapy. The mean level of serum testosterone was $0.14 \mathrm{ng} \mathrm{ml}^{-1}$ (95\% CI: $\left.0.11-0.16 \mathrm{ng} \mathrm{ml}^{-1}\right)$ at the baseline and decreased to $0.06 \mathrm{ng} \mathrm{ml}^{-1}$ ( $95 \% \mathrm{CI}: 0.02-0.09 \mathrm{ng} \mathrm{ml}^{-1}$ ) as the nadir level after the ketoconazole therapy was started. When the PSA progressed, the serum testosterone was up to $0.13 \mathrm{ng} \mathrm{ml}^{-1}(95 \%$ CI: $0.09-0.17 \mathrm{ng} \mathrm{ml}^{-1}$ ) (Figure 1). The Spearman's coefficient was $0.866(P=0.003)$. Furthermore, in the PSA non-responder group, there were six patients whose testosterone did not decrease. However, all patients in the PSA responder group had a testosterone decline.

Among the 163 enrolled patients, the most common side effect was abnormal liver function, which was observed in 15 patients $(9.2 \%)$. Other common toxicities included abnormal renal function $(8.6 \%)$, acratia $(6.1 \%)$, nausea $(3.7 \%)$, anorexia $(3.1 \%)$, vomiting $(2.5 \%)$ and declining white blood cell count $(2.5 \%)$. Three patients $(1.8 \%)$ had grade 3 toxicity, including abnormal liver/renal function, and stopped ketoconazole therapy. Grade 4 toxicity was not observed, and there were no deaths due to the side effects of the ketoconazole therapy. 
Table 1 Patient clinical and characteristics

\begin{tabular}{|c|c|c|c|}
\hline Characteristics & Number of subjects & $\begin{array}{l}\text { Median progression-free } \\
\text { survival (month) }\end{array}$ & $\mathrm{P}$ value \\
\hline Age at ketoconazole therapy, median 71 years (range 50-83 years) & & & 0.213 \\
\hline$<71$ years & 79 & 3.0 & \\
\hline$\geqslant 71$ years & 84 & 2.6 & \\
\hline Clinical T stage & & & 0.239 \\
\hline $\mathrm{T} 2$ & 66 & 3.0 & \\
\hline T3-4 & 33 & 2.8 & \\
\hline Unknown & 64 & 2.0 & \\
\hline Metastasis site & & & 0.778 \\
\hline Lymph nodes only & 10 & 3.0 & \\
\hline Bone only & 132 & 2.7 & \\
\hline Lymph nodes and bone & 16 & 2.6 & \\
\hline Internal organ & 5 & 2.8 & \\
\hline Biopsy Gleason score & & & 0.041 \\
\hline $4-6$ & 72 & 3.1 & \\
\hline 7 & 70 & 1.9 & \\
\hline $8-10$ & 21 & 3.0 & \\
\hline Nadir PSA & & & $<0.001$ \\
\hline$<0.2 \mathrm{ng} \mathrm{ml}^{-1}$ & 70 & 3.5 & \\
\hline$\geqslant 0.2 \mathrm{ng} \mathrm{ml}^{-1}$ & 93 & 1.4 & \\
\hline PSADT & & & 0.025 \\
\hline$<2.0$ months & 62 & 1.9 & \\
\hline$\geqslant 2.0$ months & 101 & 3.0 & \\
\hline Baseline PSA & & & 0.615 \\
\hline$<20 \mathrm{ng} \mathrm{ml}^{-1}$ & 76 & 2.8 & \\
\hline$\geqslant 20 \mathrm{ng} \mathrm{ml}^{-1}$ & 87 & 2.6 & \\
\hline Baseline testosterone & & & $<0.001$ \\
\hline$<0.1 \mathrm{ng} \mathrm{m}^{-1}$ & 76 & 1.6 & \\
\hline$\geqslant 0.1 \mathrm{ng} \mathrm{ml}^{-1}$ & 87 & 3.1 & \\
\hline Baseline haemoglobin & & & 0.004 \\
\hline Normal $\left(\geqslant 120 \mathrm{~g} \mathrm{I}^{-1}\right)$ & 93 & 2.8 & \\
\hline Less than normal & 70 & 1.9 & \\
\hline Baseline alkaline phosphatase & & & 0.004 \\
\hline Normal $\left(\leqslant\left. 130||^{\prime}\right|^{-1}\right)$ & 66 & 3.0 & \\
\hline Greater than normal & 97 & 1.9 & \\
\hline Docetaxel chemotherapy & & & 0.517 \\
\hline Yes & 11 & 2.0 & \\
\hline No & 152 & 2.6 & \\
\hline
\end{tabular}

Abbreviations: PSA, prostate-specific antigen; PSADT, PSA doubling time.

The cutoffs for the nadir PSA and PSADT were based on the literature; the cutoffs for the baseline PSA and testosterone were based on the Youden index; the cutoffs for the baseline haemoglobin and alkaline phosphatase were the limiting value of the normal level.

The $P$ value was calculated by the univariate Cox regression analysis.

\section{Response to therapy and predictors of PFS}

At the end of the follow-up, 160 patients experienced a PSA increase and disease progression after the ketoconazole therapy. The median PFS for all the patients was 2.6 months (0.5-8.6 months), and 51 (31.9\%) of 160 patients were determined to be PSA responders with a PSA decline of $\geqslant 50 \%$. In the PSA responders, the PFS was 3.7 months (2.7-8.6 months), and in the PSA non-responders, the PFS was only 1.7 months $(0.5-3.7$ months $)(P<0.001)$. The hazard rate (HR) was 0.138 (95\% CI: $0.085-0.223)$. The risk ratios of all the variables for PFS are described in Figure 2.

Based on the univariate and multivariate Cox regression analyses, four variables, nadir PSA, baseline haemoglobin, baseline testosterone and PSADT, were finally identified as the predictors of PFS for these 160 patients (Table 2). The nadir PSA was the strongest survival determinant among these variables, with a median PFS of 3.5 months for those with $<0.2 \mathrm{ng} \mathrm{ml}^{-1}$ and of 1.4 months for those with $\geqslant 0.2 \mathrm{ng} \mathrm{ml}^{-1}$. The patients with a baseline testosterone of $\geqslant 0.1 \mathrm{ng} \mathrm{ml}^{-1}$ had a median PFS of 3.1 months, and those with $<0.1 \mathrm{ng} \mathrm{ml}^{-1}$ had a median PFS of 1.6 months. The median PFSs for the patients who had a baseline haemoglobin of $\geqslant 120$ and $<120 \mathrm{~g} \mathrm{l}^{-1}$ were 2.8 and 1.9 months, respectively. The median PFSs for patients with a PSADT of $\geqslant 2.0$ and $<2.0$ months were 3.0 and 1.9 months, respectively.

\section{The risk model of PFS for ketoconazole therapy}

We constructed a risk model that used the four predictors. For each of the four risk factors (nadir PSA $\geqslant 0.2 \mathrm{ng} \mathrm{ml}^{-1}$, baseline testosterone $<0.1 \mathrm{ng} \mathrm{ml}^{-1}$, baseline haemoglobin less than normal $\left(<120 \mathrm{~g} \mathrm{l}^{-1}\right)$ and PSADT $<2.0$ months $)$, we divided the patients into three subgroups, which were low risk (0-1 factor), moderate risk ( 2 factors) and high risk ( $3-4$ factors). In the cohort, the PSA response rates for these three classes were $64.6 \%$ (42/65), $23.7 \%$ (9/ $38)$ and $0(0 / 57)$, respectively. The PFSs were 3.6 months (0.9-8.6 months), 3.0 months (0.5-3.7 months) and 1.4 months (0.7-3.7 months), respectively $(\mathrm{HR}=1.619, P<0.001)$ (Table 3$)$. The additional analyses about the survival probability of the risk model are further described in Figure 3. 


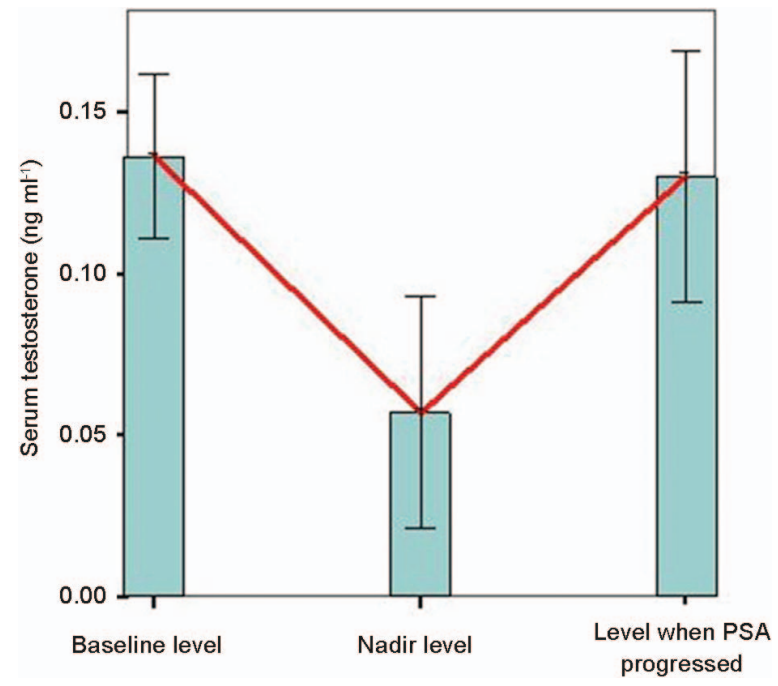

Figure 1 The change in the serum testosterone. The mean serum testosterone was $0.14 \mathrm{ng} \mathrm{ml}^{-1}$ at the baseline level, and it decreased to a nadir level of $0.06 \mathrm{ng} \mathrm{ml}^{-1}$ after the ketoconazole therapy. When the PSA progressed, the serum testosterone increased to $0.13 \mathrm{ng} \mathrm{ml}^{-1}$. The red line denotes the change in serum testosterone, and the minor axis shows the 95\% confidence interval for serum testosterone. PSA, prostate-specific antigen.

\section{DISCUSSION}

When the initial hormonal therapy has failed and the disease has progressed to CRPC, only limited options are provided for these patients, and the prognosis is poor. As one of the most important adrenal testosterone inhibitors, ketoconazole is generally utilized with

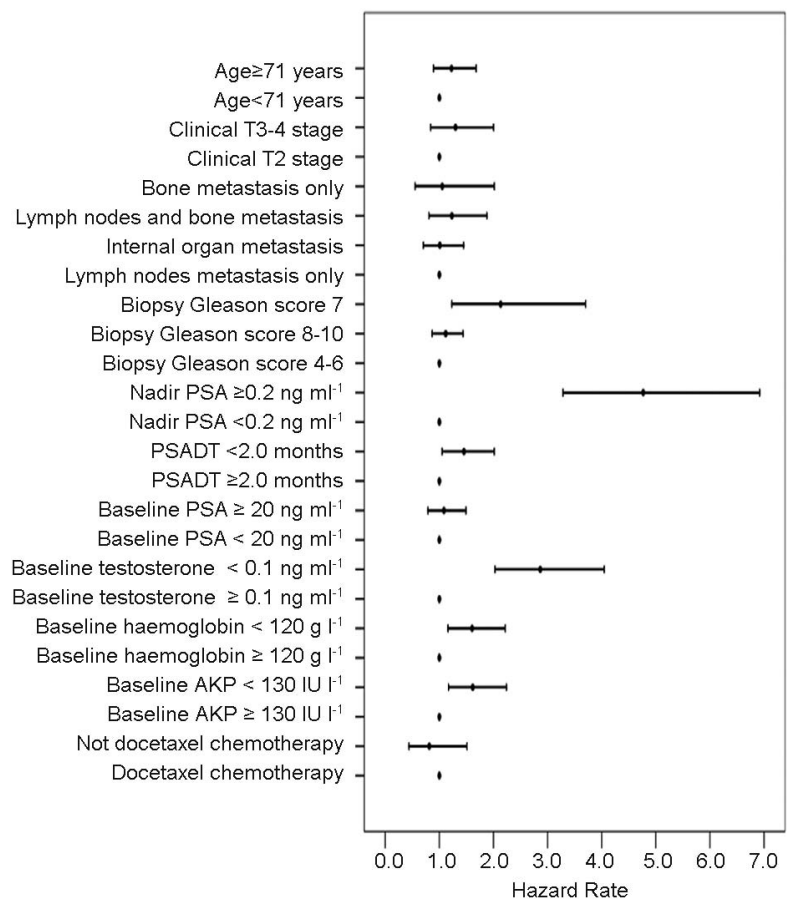

Figure 2 The risk ratios of all variables for PFS in the patients with metastatic castration-resistant prostate cancer treated by ketoconazole therapy. The red point denotes the hazard rate, and the minor axis shows the hazard rate $95 \%$ confidence interval. AKP, alkaline phosphatase; PFS, progression-free survival; PSA, prostate-specific antigen; PSADT, PSA doubling time.
Table 2 The predictors in the multivariate Cox regression analysis for PFS

\begin{tabular}{|c|c|c|c|c|}
\hline Variables & Level & $H R$ & $H R(95 \% \mathrm{Cl})$ & P value \\
\hline Nadir PSA (ng ml ${ }^{-1}$ ) & $<0.2$ vs. $\geqslant 0.2$ & 3.700 & $1.802-7.593$ & $<0.001$ \\
\hline $\begin{array}{l}\text { Baseline testosterone } \\
\left(\mathrm{ng} \mathrm{ml}^{-1}\right)\end{array}$ & $\geqslant 0.1$ vs. $<0.1$ & 3.644 & $1.785-7.442$ & $<0.001$ \\
\hline $\begin{array}{l}\text { Baseline haemoglobin } \\
\left(\mathrm{g}^{-1}\right)\end{array}$ & $\geqslant 120$ vs. $<120$ & 2.333 & $1.363-3.994$ & 0.002 \\
\hline PSADT (months) & $\geqslant 2.0$ vs. $<2.0$ & 1.982 & $1.078-3.646$ & 0.028 \\
\hline
\end{tabular}

Abbreviations: $\mathrm{Cl}$, confidence interval; $\mathrm{HR}$, hazard rate; PFS, progression-free survival; PSA, prostate-specific antigen; PSADT, PSA doubling time.

All variables were entered into the multivariate Cox regression analysis for PFS. The HR of the baseline testosterone, nadir PSA, baseline haemoglobin and PSADT were the highest values for these variables, and the $P$ values were less than 0.05 only for these four factors.

hydrocortisone as a subsequent hormonal therapy for patients with CRPC. $^{7,9,11}$ The outcome seems to have a moderate benefit with a PSA response rate of $30 \%-60 \%$ and a PFS of 3-9 months. In this study, we analysed the prognosis for Chinese patients receiving ketoconazole therapy. The median PFS was 2.6 months with a PSA response rate of $31.9 \%$, which were similar to other studies.

Furthermore, we noted that not all the patients would have an ideal survival benefit from the ketoconazole therapy in different studies. In fact, the median PFS seemed to exhibit a large disconnect, which was from more than 8 months to less than 3 months. ${ }^{7,9}$ Some scholars suggested that a possible reason for this discrepancy was a selection bias. ${ }^{11}$ However, this explanation seemed to be farfetched. If all the patients with CRPC were given this management strategy, it may increase the non-essential treatment expense and delay the subsequent treatment options. Thus, who can benefit from ketoconazole therapy? Are there any predictors for ketoconazole effective treatment? Is there any pertinent risk model of the survival benefit for these patients in the clinical practice?

This study focussed on a predictor analysis of PFS and established a risk model for ketoconazole effective treatment. According to the univariate and multivariate Cox regression analyses, the nadir PSA, baseline testosterone, baseline haemoglobin and PSADT were certified as independent predictors of ketoconazole effective treatment.

The nadir PSA was shown to be the strongest survival determinant compared with the other variables. The risk of progression for patients with a nadir PSA of $\geqslant 0.2 \mathrm{ng} \mathrm{ml}^{-1}$ was four times greater than that for patients with a nadir PSA of $<0.2 \mathrm{ng} \mathrm{ml}^{-1}$, and the median PFS was 2 months longer in the latter. In fact, the nadir PSA was a strong prognostic factor in various studies. Kobayashi et al. ${ }^{20}$ reported that the nadir PSA in first-line therapy was a possible surrogate for the responsiveness to the second-line therapy and that the survival for those patients with a nadir PSA of $\geqslant 0.2 \mathrm{ng} \mathrm{ml}^{-1}$ was significantly shorter than the others. Miyamoto et al. ${ }^{21}$ reported that the nadir PSA after the first-line and second-line hormonal treatments was an independent prognostic factor for the 3-and 5-year overall survival for metastatic prostate cancer. In our study, the baseline haemoglobin and PSADT were also identified as predictors for ketoconazole therapy, and they had been proven to be associated with prognosis in the literature. $^{22-25}$

The change in the serum testosterone during the ketoconazole therapy showed a positive correlation between the serum testosterone and PSA levels by Spearman's rank correlations. When the PSA declined, the serum testosterone always decreased; in contrast, when PSA increased, the serum testosterone would increase. In this study, we 
Table 3 PFS and PSA response rate performance of the risk model in this study

\begin{tabular}{llccc}
\hline Risk group & No. (\%) & PSA response rate (\%) & Median PFS (months) & HR (95\% Cl) \\
\hline Low risk & $65(40.6)$ & $42 / 65(64.6)$ & 3.6 & $1.619(1.444-1.815)$ \\
Moderate risk & $38(23.8)$ & $9 / 38(23.7)$ & 3.0 & Reference \\
High risk & $57(35.6)$ & $0 / 57(0)$ & 1.4 & $<0.001$ \\
\hline
\end{tabular}

Abbreviations: $\mathrm{Cl}$, confidence interval; HR, hazard rate; PFS, progression-free survival; PSA, prostate-specific antigen; PSADT, PSA doubling time.

Low risk denotes patients with $0-1$ risk factor, moderate risk denotes 2 risk factors and high risk denotes $3-4$ risk factors. The risk factors included a nadir PSA of $\geqslant 0.2$ ng $\mathrm{ml}^{-1}$, a baseline testosterone of $<0.1 \mathrm{ng} \mathrm{ml}^{-1}$, a baseline haemoglobin less than normal $\left(<120 \mathrm{~g} \mathrm{I}^{-1}\right.$ ) and a PSADT of $<2$ months. The PSA response rate was defined as the percentage of patients with a PSA decline of $\geqslant 50 \%$ compared with the baseline PSA level during the ketoconazole therapy.

found the existence of a serum testosterone dynamic fluctuation that was relative to the PSA change during the ketoconazole therapy; Figure 2 shows a diagram of the serum testosterone that helps to evaluate the changing trend. These data suggested that some regularity in the serum testosterone level existed for most patients, and the Cox regression analysis suggested that a high-baseline serum testosterone $\left(\geqslant 0.1 \mathrm{ng} \mathrm{ml}^{-1}\right)$ was positively associated with a longer PFS. A probable mechanism of action for ketoconazole is via the blockade of the conversion of lanosterol to cholesterol by inhibiting CYP17 and subsequently inhibiting adrenal testosterone. ${ }^{16}$ Thus, an equally plausible explanation was that when the serum testosterone was at the castration level $\left(\leqslant 0.5 \mathrm{ng} \mathrm{ml}^{-1}\right)$, the higher the serum testosterone level, the higher the fraction of the serum testosterone from the adrenal gland. Thus, patients with a high serum testosterone, but still within the castration level, would have the maximum clinical benefit from inhibiting adrenal testosterone by ketoconazole therapy.

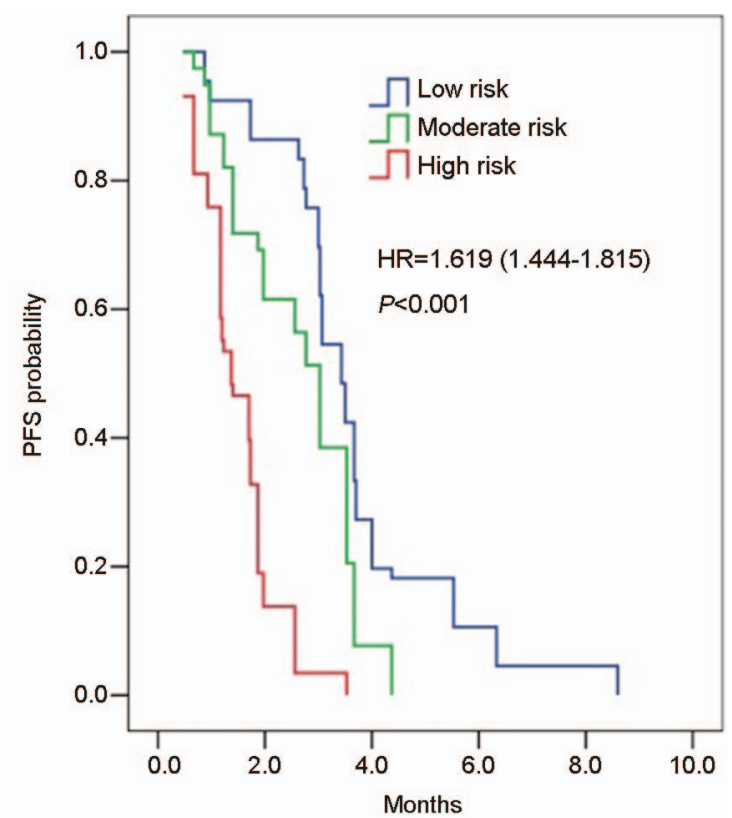

$\begin{array}{rrrrrrr}\text { Patient Number at subgroup } & & & & \\ \text { Low risk } & 65 & 57 & 18 & 7 & 3 & 0 \\ \text { Moderate risk } & 38 & 24 & 3 & 0 & 0 & 0 \\ \text { High risk } & 57 & 8 & 0 & 0 & 0 & 0\end{array}$

Figure 3 A comparison of the PFS curves among the low-, moderate- and highrisk groups by the log-rank test $(H R=1.619, P<0.001)$. The median PFS was 3.6 months in the low-risk group, which was longer than in the other two groups, which had PFSs of 3.0 and 1.4 months (low risk vs. moderate risk: $H R=1.364$, $P=0.004$; low risk vs. high risk: $\mathrm{HR}=1.656, P<0.001$; moderate risk vs. high risk: $\mathrm{HR}=1.834, P<0.001$ ). $\mathrm{HR}$, hazard rate; PFS, progression-free survival.
In this study, a risk model constructed using four prognostic factors was used to stratify patients into risk classes for prognosis in the clinical practice. Using the risk model, a patient in the low-risk group with none or only one risk factor has the best survival benefit, with a longer median PFS and a higher PSA response rate, compared with the patients in the high-risk groups. The risk model could be applied to patient selection for ketoconazole therapy and may be useful to help redefine a new standard care for patients with MCRPC in the future clinical trials, such as for abiraterone.

Several limitations existed in our study. First, it had a limited number of subjects. However, this limitation was shared with other studies that have developed other useful predictive tools. Second, the PFS in the group with a biopsy Gleason score of 7 was shorter than that of the group with a biopsy Gleason score of 8-10, and the smaller number of the latter may be responsible for the result. Third, the risk model was not externally validated for discrimination, and thus, we need further studies or results from other scholars' studies to confirm these results.

\section{CONCLUSIONS}

Our study indicated that ketoconazole was a moderate activity agent for patients with mCRPC and demonstrated a risk model including the nadir PSA, baseline haemoglobin, baseline testosterone and PSADT as a tool for patient selection and prognosis evaluation. Currently, abiraterone, a new CYP17 inhibitor, had begun to be used to treat mCRPC. ${ }^{26,27}$ Given the similar mechanisms of abiraterone and ketoconazole, the present study may supply some suggestions for further studies on abiraterone in the future.

\section{AUTHOR CONTRIBUTIONS}

GWL and XDY contributed to the conception and design of the study, participated in the analysis and interpretation of the data and drafted the manuscript. DWY supplied all the patient data and approved the final manuscript. YZ participated in the statistical analysis and interpretation of the data. SLZ, BD, HLZ, YJS and CGM participated in the collection of the data.

\section{COMPETING FINANCIAL INTERESTS}

The authors declare no competing financial interests.

\section{ACKNOWLEDGMENTS}

The work was supported by the National Natural Science Foundation of China (No. 30973009) and the Shanghai Natural Science Foundation

(No. 11ZR1407400).

1 Prostate Cancer Trialists' Collaborative Group. Maximum androgen blockade in advanced prostate cancer: an overview of the randomised trials. Lancet 2000; 355: 1491-8.

2 Schmitt B, Wilt TJ, Schellhammer PF, DeMasi V, Sartor 0 et al. Combined androgen blockade with nonsteroidal antiandrogens for advanced prostate cancer: a systematic review. Urology 2001; 57: 727-32. 
3 Nakabayashi M, Oh WK, Jacobus S, Regan MM, Taplin ME et al. Activity of ketoconazole after taxane-based chemotherapy in castration-resistant prostate cancer. BJU Int 2010; 105: 1392-6.

4 Oh WK. Secondary hormonal therapies in the treatment of prostate cancer. Urology 2002; 60: 87-92.

5 Suzuki H, Okihara K, Miyake H, Fujisawa M, Miyoshi S et al. Alternative nonsteroidal antiandrogen therapy for advanced prostate cancer that relapsed after initial maximum androgen blockade. J Urol 2008; 180: 921-7.

6 Attard G, Reid AH, Yap TA, Raynaud F, Dowsett M et al. Phase I clinical trial of selective inhibitor of CYP17, abiraterone acetate, confirms that castration-resistant prostate cancer commonly remains hormone driven. J Clin Oncol 2008; 26: 456371.

7 Nakabayashi M, Xie W, Regan MM, Jackman DM, Kantoff PW et al. Response to low dose ketoconazole and subsequent dose escalation to high-dose ketoconazole in patients with androgen-independent prostate cancer. Cancer 2006; 107: 975-81.

8 Procopio G, Guadalupi V, Giganti MO, Mariani L, Salvioni R et al. Low dose of ketoconazole in patients with prostate adenocarcinoma resistant to pharmacological castration. BJU Int 2011; 108: 223-7.

9 Harris KA, Weinberg V, Bok RA, Kakefuda M, Small EJ. Low dose ketoconazole with replacement doses of hydrocortisone in patients with progressive androgen independent prostate cancer. J Urol 2002; 168: 542-5.

10 Figg WD, Liu Y, Arlen P, Gulley J, Steinberg SM et al. A randomized, phase II trial of ketoconazole plus alendronate versus ketoconazole alone in patients with androgen independent prostate cancer and bone metastases. J Urol 2005; 173: 790-6.

11 Small EJ, Halabi S, Dawson NA, Stadler WM, Rini BI et al. Antiandrogen withdrawal alone or in combination with ketoconazole in androgen-independent prostate cancer patients: a phase III trial (CALGB 9583). J Clin Oncol 2004; 22: 1025-33.

12 Figg WD, Woo S, Zhu W, Chen X, Ajiboye AS et al. A phase I clinical study of high dose ketoconazole plus weekly docetaxel for metastatic castration resistant prostate cancer. J Urol 2010; 183: 2219-26.

13 Eklund J, Kozloff M, Vlamakis J, Starr A, Mariott M et al. Phase II study of mitoxantrone and ketoconazole for hormone-refractory prostate cancer. Cancer 2006; 106: 2459 65.

14 Ryan CJ, Halabi S, Ou SS, Vogelzang NJ, Kantoff P et al. Adrenal androgen levels as predictors of outcome in prostate cancer patients treated with ketoconazole plus antiandrogen withdrawal: results from a cancer and leukemia group B study. Clin Cancer Res 2007; 13: 2030-7.

15 Harris KA, Weinberg V, Bok RA, Kakefuda M, Small EJ. Low dose ketoconazole with replacement doses of hydrocortisone in patients with progressive androgen independent prostate cancer. J Urol 2002; 168: 542-5.
16 Bubley GJ, Carducci M, Dahut W, Dawson N, Daliani D et al. Eligibility and response guidelines for phase II clinical trials in androgen-independent prostate cancer: recommendations from the Prostate-Specific Antigen Working Group. J Clin Oncol 1999; 17: 3461-71.

17 Hussain M, Tangen CM, Higano C, Schelhammer PF, Faulkner J et al. Absolute prostate-specific antigen value after androgen deprivation is a strong independent predictor of survival in new metastatic prostate cancer: data from Southwest Oncology Group Trial 9346 (INT-0162). J Clin Oncol 2006; 24: 3984-90.

18 Lin GW, Yao XD, Zhang SL, Dai B, Ma CG et al. Prostate-specific antigen half-life: a new predictor of progression-free survival and overall survival in Chinese prostate cancer patients. Asian J Androl 2009; 11: 443-50.

19 Schisterman EF, Perkins NJ, Liu A, Bondell H. Optimal cut-point and its corresponding Youden Index to discriminate individuals using pooled blood samples. Epidemiology 2005; 16: 73-81.

20 Kobayashi M, Suzuki K, Kurokawa S, Yuzawa M, Morita T. Second-line antiandrogen therapy in Japanese men with advanced prostate cancer relapsed after primary combined androgen blockade: who will benefit from second-line hormonal therapy? Urol Int; e-pub ahead of print 10 August 2010; doi:10.1159/000318056

21 Miyamoto S, Ito K, Miyakubo M, Suzuki R, Yamamoto T et al. Impact of pretreatment factors, biopsy Gleason grade volume indices and post-treatment nadir PSA on overal survival in patients with metastatic prostate cancer treated with step-up hormona therapy. Prostate Cancer Prostatic Dis 2012; 15: 75-86.

22 Beer TM, Tangen CM, Bland LB, Hussain M, Goldman BH et al. The prognostic value of hemoglobin change after initiating androgen-deprivation therapy for newly diagnosed metastatic prostate cancer: A multivariate analysis of Southwest Oncology Group Study 8894. Cancer 2006; 107: 489-96.

23 Klayton TL, Ruth K, Buyyounouski MK, Uzzo RG, Wong YN et al. PSA doubling time predicts for the development of distant metastases for patients who fail 3DCRT or IMRT using the Phoenix definition. Pract Radiat Oncol 2011; 1: 235-42.

24 Keizman D, Huang P, Antonarakis ES, Sinibaldi V, Carducci MA et al. The change of PSA doubling time and its association with disease progression in patients with biochemically relapsed prostate cancer treated with intermittent androgen deprivation. Prostate 2011; 71: 1608-15.

25 Teeter AE, Presti JC Jr, Aronson WJ, Terris MK, Kane CJ et al. Does PSADT after radica prostatectomy correlate with overall survival?-A report from the SEARCH database group. Urology 2011; 77: 149-53.

26 Pal SK, Sartor O. Current paradigms and evolving concepts in metastatic castrationresistant prostate cancer. Asian J Androl 2011; 13: 683-9.

27 Tagawa ST, Beltran H. Abiraterone plus prednisone improves survival in metastatic castration-resistant prostate cancer. Asian J Androl 2011; 13: 785-6. 